\title{
Reorganization of the injured brain: implications for studies of the neural substrate of cognition
}

\author{
Jesper Mogensen* \\ The Unit for Cognitive Neuroscience, Department of Psychology, University of Copenhagen, Copenhagen, Denmark
}

\section{Edited by:}

Morten Overgaard, Aarhus University,

Denmark

\section{Reviewed by:}

Gerald Zernig, Medical University

Innsbruck, Austria

\section{*Correspondence:}

Jesper Mogensen, Department of Psychology, The Unit for Cognitive Neuroscience, University of

Copenhagen, Oester Farimagsgade 2A, DK-1353 Copenhagen K, Denmark. e-mail: jesper.mogensen@psy.ku.dk
In the search for a neural substrate of cognitive processes, a frequently utilized method is the scrutiny of post-traumatic symptoms exhibited by individuals suffering focal injury to the brain. For instance, the presence or absence of conscious awareness within a particular domain may, combined with knowledge of which regions of the brain have been injured, provide important data in the search for neural correlates of consciousness. Like all studies addressing the consequences of brain injury, however, such research has to face the fact that in most cases, post-traumatic impairments are accompanied by a "functional recovery" during which symptoms are reduced or eliminated. The apparent contradiction between localization and recovery, respectively, of functions constitutes a problem to almost all aspects of cognitive neuroscience. Several lines of investigation indicate that although the brain remains highly plastic throughout life, the posttraumatic plasticity does not recreate a copy of the neural mechanisms lost to injury. Instead, the uninjured parts of the brain are functionally reorganized in a manner which - in spite of not recreating the basic information processing lost to injury - is able to allow a more or less complete return of the surface phenomena (including manifestations of consciousness) originally impaired by the trauma. A novel model [the Reorganization of Elementary Functions-model] of these processes is presented - and some of its implications discussed relative to studies of the neural substrates of cognition and consciousness.

Keywords: neural plasticity, reorganization, brain injury, localization of function, recovery, recovery of function, consciousness, neural organization

\section{INTRODUCTION}

Research into the relationship between on the one hand brain injury [be it vascular or traumatic (TBI)] and on the other hand various cognitive processes remains a crucial part of cognitive neuroscience. Some of the consciousness-related phenomena studied in brain injured patients are hemispatial neglect and blindsight. There is a constantly growing body of clinical as well as experimental data on various types of neglect and blindsight in focally lesioned patients (e.g., Natsoulas, 1997; Marcel, 1998; Rossetti et al., 1998; Kentridge et al., 1999; Danckert and Goodale, 2000; Schindler et al., 2006; Bartolomeo, 2007; Silvanto et al., 2008; Funk et al., 2010). Even within other areas than visual perception lesion-induced dissociations between conscious and non-conscious processes are being addressed in attempts to yield information regarding the neural processes mediating subjective consciousness (e.g., Lane et al., 1997). Studies focusing on the manifestations of consciousness in brain injured patients fall within the framework of research addressing "localization of functions" within the brain. Other cognitive domains exhibit a constantly growing body of such studies, e.g., language (e.g., Thomas et al., 1997; Thulborn et al., 1999; Ansaldo et al., 2002; Ansaldo and Arguin, 2003; Perani et al., 2003; Baumgaertner et al., 2005; Meinzer et al., 2008; Specht et al., 2009; Szaflarski et al., 2010). Associating various functions with specific brain regions is one of the main research traditions of cognitive neuroscience. But it is also an endeavor facing both methodological and theoretical challenges (e.g., Mogensen and Malá, 2009) not the least the apparent contradiction between the localization and post-traumatic recovery, respectively, of various "functions." Focusing on the recently developed Reorganization of Elementary Functions (REF)-model (Mogensen and Malá, 2009) the present communication discusses these issues with respect to the understanding of the neural substrate and post-traumatic recovery of cognitive functions in general. A subsequent paper (Overgaard and Mogensen, 2011) will deal more specifically with the issue of what is required in terms of theoretical conceptualization as well as experimental documentation if post-traumatic functional recovery is to be taken as documentation of multiple realizations of neural substrates of consciousness.

\section{LOCALIZATION AND/OR RECOVERY}

There can be little doubt that the brain is regionally specialized. Various brain structures and substructures perform apparently unique types of information processing and consequently participate differentially in the mediation of various types of behavior and cognition. In other and more commonly used words: the functions of the brain are regionally localized. The main support for this assumption grows out of two research traditions. One of these is the study of post-traumatic impairments in brain injured individuals - patients or experimental animals. In such studies, the profile of post-injury symptoms is compared to the localization and extent of brain injury. The logics of these lesion experiments (see e.g., Coltheart, 2001; Selnes, 2001; controlled experiments in animal models and clinical studies in brain injured patients) is that in the absence of a brain structure, the symptoms must reflect 
the absence of functional contributions from the affected neural machinery - and that with the proper analysis and comparisons across symptoms, conclusions can be drawn regarding the information processing of the missing brain circuitry. A more recent contribution to this type of study is the use of transcranial magnetic stimulation (e.g., Pascual-Leone et al., 1999, 2000; Walsh and Cowey, 2000) in which the functional integrity of a part of the brain is temporarily disturbed - allowing an analysis of the consequences of what can be seen as a "temporary lesion." The other main source of support for functional localization within the brain is the studies utilizing various types of neuroimaging techniques. By studying the regional pattern of brain activation during the performance of various tests, one can - provided adequate baseline measures are utilized for the "subtraction" from the test condition - provide information about whether or not a particular brain structure changes its level of activity (often reflected directly or indirectly as a change in metabolism and blood supply) in association with the performance of a particular task or stimulation. Numerous contradictions exist within these branches of the neuroscientific literature, but there is an overall agreement that although often poorly understood, there is a regional functional specialization within the brain - a "functional localization" (e.g., Monakow, 1914; Coltheart, 2001; Selnes, 2001; Kringelbach and Rolls, 2004).

As mentioned above, a crucial aspect of the interpretation of results from various types of "lesion experiments" is that in the absence of a brain structure - and consequently the functional contributions from that circuitry - the behavior and conscious manifestations of the individual must reflect the lack of whatever functional contributions were provided by the now missing part of the brain. But if it is assumed that the lost circuitry is posttraumatically never regained (an issue to which I will return later), one should expect the impairments after brain injury to be chronic. Nevertheless, it is a well-established fact that a post-traumatic functional recovery does occur.

In patients as well as in animal models of brain injury, most lesion-associated impairments post-traumatically undergo some level of "recovery" - at least within cognitive domains not closely linked to the direct in- and output pathways. Less or even no recovery may be seen within some sensory and motoric domains. But for almost all "higher" cognitive functions, trauma-related impairments are followed by an apparent return toward the proficiency seen pre-traumatically (e.g., Ramachandran and Blakeslee, 1998; Carney et al., 1999; Buller and Hardcastle, 2000; Panksepp and Panksepp, 2000; León-Carrión and Machuca-Murga, 2001; Mogensen et al., 2004, 2007; Mogensen and Malá, 2009; Rohling et al., 2009). In most cases, such a functional recovery is associated with more or less formalized and institutionalized rehabilitative training, but in the absence of such training, "spontaneous" recovery is also seen (e.g., León-Carrión and Machuca-Murga, 2001). It must, however, be remembered that even in the absence of a formalized post-traumatic training program, practically all brain injured organisms (patients and experimental animals alike) are subjected to the informal training of daily life activities. Even the most basic daily activities and communicative efforts constitute challenges and tasks which the brain injured individual must attempt to meet and master. Consequently, the absence of an externally imposed training regime does not allow a claim that the potential occurrence of a functional recovery is independent of interactions with the environment. Some instances in which a "spontaneous" recovery may be of a more automatic nature can, however, be seen in cases where a lesion-induced "penumbra" gradually disappears. The "penumbra" phenomenon can briefly be described as a situation in which injury within one part of the brain causes other brain areas to receive a reduced level of blood supply. While being sufficient for the survival of neurons within that penumbra region, the reduced blood supply does not allow a normal level of functionality. Consequently, the observable symptoms are not only associated with the trauma per se but also with the impaired neural activities within the penumbra. Penumbras, however, mostly disappear spontaneously and allow a return to normal levels of functional performance within that region of the brain (e.g., Choi et al., 2007).

Although sometimes incomplete, this post-traumatic functional recovery may both clinically and in animal models turn out to be "complete" - defined as the acquisition of a post-traumatic proficiency equal to that seen in the absence of any brain injury (e.g., Mogensen et al., 2004). In case of animal models, this is even seen under circumstances ensuring the complete removal of the brain structure in question as well as a well-established pre-traumatic functional baseline.

If the relatively few instances in which post-traumatic symptoms can be associated with penumbras or similar phenomena are excluded, what remains is the apparent contradiction between the two phenomena of "functional localization" and "recovery of function."

A radical and maybe tempting way to utilize lesion experiments without having to deal with the contradiction between localization and recovery of functions is to accept only the post-traumatic symptoms as relevant to arguments regarding functional specialization, in case those symptoms turn out to be "chronic" - never to demonstrate any functional recovery. Such a radical position has been advocated by Olton (1978). It has to be realized, however, that if only those instances in which post-traumatic impairments persist chronically are to be considered when conclusions are drawn from lesion experiments, the vast majority of such clinical and animal model derived data would have to be discarded.

But the arguments against dismissing all but the chronic posttraumatic symptoms for consideration when the principles of localization are considered, are not only of such a practical nature (although disregarding almost the entire mass of post-traumatic data is in itself not an insignificant obstacle!). The fact that a functional recovery can actually take place in spite of the continued presence of a lesion, which originally had such an impact on the information processing of the brain that significant symptoms occurred, is in itself a highly relevant phenomenon. It indicates dynamic changes, which must be an essential part of the functional organization of the brain. If these phenomena are not considered in neuroscientific studies and the construction of various models, the result will be an incomplete and lacking understanding of the functionally dynamic brain.

But in which ways, then, can one imagine the apparent return (potentially to a normal level of proficiency) of the behavioral and cognitive abilities of a brain injured organism? One possibility is that post-traumatically the brain is able to reconstruct - within the region of injury or elsewhere - a circuitry, which can accomplish 
an information processing similar to what has been lost to injury. Although it is well known that the actual site of injury mostly turn into scar tissue, this does not in itself preclude the possibility of a recreation somewhere in the brain of circuitry fulfilling the same information processing demands as the lost structure.

\section{IS THE BASIC CIRCUITRY OF THE BRAIN RECREATED POST-TRAUMATICALLY?}

In order to evaluate the likeliness that a reconstruction of the lost circuitry can occur in the injured brain, it is relevant to compare the types of plasticity available in the injured adult brain to the plastic processes, which contribute to the original construction of such networks during development.

When the adult brain is injured, the trauma itself induces a range of changes in gene expression - both in the tissue immediately surrounding the site of injury and in more remote parts of the brain. Some such changes are likely primarily to contribute to the detrimental effects of the injury - for instance by promoting metabolic dysfunction, inflammatory responses, etc. (e.g., Delfs et al., 1995; Hermann et al., 1999; Harris et al., 2001; Rao et al., 2003). Other changes in gene expression are likely to be supportive of rehabilitation - promoting plasticity and reorganization (e.g., Witte, 1998; Frost et al., 2003; Kleim et al., 2003; Nudo, 2003). One such process may be the increased occurrence of long-term potentiation (LTP) like synaptic plasticity after brain injury (e.g., Hagemann et al., 1998).

Relative to the issue of recreation of circuitry similar to what has been lost to injury, it might be especially relevant that injury to the brain potentiates the ongoing neurogenesis (e.g., Magavi et al., 2000; Scharff et al., 2000; Arvidsson et al., 2002; Nakatomi et al., 2002; Chen et al., 2004). A reason for this to be important is that during maturation neurons undergo a number of changes - reducing their similarity to the developing neurons, which originally formed various circuits (e.g., Fawcett et al., 1989; Chen et al., 1995; Goldberg et al., 2002). In contrast, the newly formed neurons produced by adult neurogenesis are unlikely to have similar limitations.

If the newly created neurons in the injured brain are to reach a specific destination and contribute to recreation of a circuit, they will have to migrate in an appropriate manner. After injury, mature astrocytes are able to transform themselves into radial glial cells similar to those guiding neural migration during development (e.g., Rakic, 1971, 1985). Such radial glial cells are able to guide the migration of immature neurons even in the adult brain (e.g., Leavitt et al., 1999). Further optimism regarding the potentials of the injured adult brain may come from the fact that most substances, which played a guiding role during the original outgrowth of dendrites and axons (e.g., Keynes and Cook, 1992; Brose et al., 1999; Chen et al., 2000; Hiramoto et al., 2000; Polleux et al., 2000) are also present in the adult brain (e.g., Koeberle and Bahr, 2004). This optimism may, however, be tempered by the observation that the distribution of these substances undergoes major changes during the maturation of the brain - making it questionable whether they in an injured adult brain can play similar roles to those of development (e.g., Harel and Strittmatter, 2006). The most important factors preventing immature neurons in the adult nervous system from recreating the injured circuitry, may, however, be associated with glial cells and myelin (e.g., Berry, 1982; Schwab and Thoenen, 1985; Schäfer et al., 2008). Especially important may be the astrocyte-produced chondroitin sulfate proteoglycans (CSPGs), which play an important role in terminating the developmentally "critical" periods (e.g., Pizzorusso et al., 2002; Berardi et al., 2004; McGee et al., 2005). While consolidating the plastic processes occurring during critical periods, these substances may also play a role, which prevents an adult recreation of the circuit lost to injury (e.g., Del Rio and Soriano, 2007; Schäfer et al., 2008). An improved functional recovery (potentially associated with recreation of lost circuitry) has been found when the CSPGs are pharmacologically inhibited locally (e.g., Del Rio and Soriano, 2007). While such a local inhibition may have therapeutic potentials in the future, the results also demonstrate that without an external intervention, the CSPGs are likely to prevent or at least reduce the possibility of a post-traumatic re-establishment of the circuitry and thereby information processing lost to trauma.

\section{THE MECHANISMS OF POST-TRAUMATIC FUNCTIONAL RECOVERY}

While it is important in these ways to establish whether the posttraumatically available neuroplasticity seems capable of processes, which can create a copy of what has been lost - another and at least as important approach is to scrutinize the neural and cognitive processes accompanying the actual functional recovery. An extensive animal model-based research program (e.g., Mogensen et al., 2002, 2003, 2004, 2005, 2007) has performed such an analysis and some of the results have been reviewed by Mogensen and Malá (2009). What has emerged is a pattern of principles regarding the mechanisms mediating post-traumatic recovery. Three general principles are especially important - describing the situation after a successful post-traumatic rehabilitation:

1. Modification of degree of contribution to task mediation by individual brain structures

- Some structures exhibit an increased or decreased level of contribution to task mediation.

2. Task dependent and dissimilar neural substrates

- After a given lesion, the functional recovery of various cognitive tasks is mediated by unique and dissimilar neural substrates.

3. Application of new cognitive strategies

- The fully post-traumatically recovered individuals solve the task by applying new strategies that are dissimilar to those applied pre-traumatically.

These three principles - like the above consideration of the types of plasticity available in the developing and injured, mature brain, respectively - indicate that a recreation of the lost circuitry is unlikely. Both the second and third of the above principles show that the neural mechanisms mediating post-traumatic functional recovery do not include a copy of what has been lost to trauma. If any part of the injured brain at the end of rehabilitation training contained a circuitry similar to what was pre-traumatically available, it would be expected that all cognitive domains affected by the lesion would post-traumatically receive equal contribution to functional recovery from the brain region within which the circuitry had been (re)created. This possibility, however, is contradicted by 
Principle 2. Additionally, Principle 3 contradicts the post-traumatic re-establishment of information processing identical to what was available pre-traumatically: if post-traumatic processes had re-established the information processing of the injured structure, one would expect not only task solution of a proficiency similar to that seen preoperatively, but also that such a task solution would employ similar strategies to those of the pre-traumatic situation.

But if the post-traumatic functional recovery is not mediated via mechanisms recreating what has been lost to injury, how can a sometimes even complete level of proficiency be re-established within traumatically impaired cognitive domains?

The recently proposed REF model of Mogensen and Malá (2009) is an attempt to describe neural and cognitive mechanisms, which in spite of the absence of a recreation of the lost circuitry - can account for a potentially full proficiency of post-traumatic cognitive recovery.

At the most basic level of the REF-model are the information processing modules named Elementary Functions (EFs). The EFs are truly localized in the sense that they are mediated by local circuitry within a structure of the brain. Each traditionally defined neural structure (e.g., the hippocampus or the dorsolateral prefrontal cortex) contains the neural substrates of a huge number of EFs. When a region of the brain is lost to injury, all EFs mediated by the lost tissue are - according to the REF-model - irreversibly lost, too. The information processing of an individual EF is of a highly basic and "modular" type. An EF does not in itself have any of the "functions" traditionally described by psychology. Psychologically defined functions such as object discrimination, explicit memory, or allocentric spatial orientation belong at a different level of analysis (to be described shortly). The "function" of an EF may more easily be described in mathematical terms - rather than in the vocabulary of for instance cognitive psychology. In contrast, the "functions" and cognitive domains normally described by psychology are in the REF-model represented at the third and highest level - the level of surface phenomena. The surface phenomena of the REF-model consist of observable behavior (for instance the performance of a task by a patient or an experimental animal) as well as conscious manifestations such as the subjective experience of recognizing a familiar face or planning a course of action in order to solve a problem. It is at the level of these surface phenomena that post-traumatic functional recovery is normally defined and evaluated. Tests conducted in order to determine what is impaired by brain injury address the observable behavior as well as whatever representations can be obtained of the subjective experiences of the patient. And it is at the same level that the more or less complete recovery of these impaired dimensions of cognition and consciousness are determined - using similar methods to those utilized during diagnosis. An essential component of the REF-model is the layer of analysis introduced between the basic layer of the EFs and the uppermost layer of the surface phenomena. This intermediate level consists of the processes named algorithmic strategies (ASs).

Each AS consists of numerous interacting EFs. Most - if not all - ASs are established as the result of experience and learning. Most EFs are simultaneously part of several or many ASs. While the individual EFs are strictly localized within a subregion of the brain, the neural substrate of an AS consists of the neural substrates of all its constituent EFs as well as the interconnections between the neural substrates of these EFs. This makes the neural substrate of an AS highly distributed and in most cases components of the neural substrate of an AS will be found within a number of brain structures. The information processing of an AS is the mechanism enabling a specific surface phenomenon. For instance, a specific type of solution of a task is obtained by activation of a particular AS. Most surface phenomena can be realized via the activity of multiple ASs. Unless special analytical techniques are employed, it may at the surface level not be possible to discriminate between behavioral or conscious phenomena reflecting two related but different ASs. Whenever brain injury destroys the neural substrate of one or more of the constituent EFs within an AS, that AS is lost. Consequently, the surface phenomena associated with the activity of the lost AS are also lost and post-traumatically an impairment is registered.

According to the REF-model, the mechanisms enabling a posttraumatic functional recovery are special cases of a more general mechanism, which in the intact brain has evolved as a crucial aspect of learning and problem solving. When an individual encounters a situation calling for a task solution for which there is no established procedure available, a mechanism is initiated during which various existing ASs are "tested out." A "selector/evaluator" mechanism - which resembles (without being identical to) the "Supervisory Attentional System" (SAS) of Norman and Shallice (1986) - sequentially activates existing ASs. When activated, an AS results in the associated surface phenomenon and, in turn, the quality of the resultant behavior or mental manifestation is evaluated. In case the desired result is obtained, activation of that AS will in the future be associated with the situation in question. This mechanism resembles - without being identical to - the mechanism of "hypotheses" evaluation described by Krechevsky $(1932,1933)$. In case activation of an existing AS can obtain the desired result, the neural plasticity associated with this entire process is restricted to modifications within the selector/evaluator mechanism - plasticity ensuring a future association between the situation in question and activation of the successfully selected AS.

If, however, a situation requiring the solution of a problem cannot be solved by activation of any existing AS, a novel AS will have to be established. The creation of a novel AS involves a reorganization of the functional interaction between EFs. This is the "Reorganization of Elementary Functions" process, which has given name to the REF-model. Such a reorganization (see Mogensen and Malá, 2009) utilizes a type of process resembling the backpropagation algorithm (e.g., Rumelhart and McClelland, 1986; Werbos, 1994). Such mechanisms - constantly utilizing the feedback of the environment - form a novel AS by combining a set of EFs, which previously did not constitute an interacting entity. Whenever such a REF-process is required in order to successfully obtain a task solution, the required neuroplasticity includes modified connections between the neural substrates of the constituent EFs. Additionally, the complete process of eventual activation of the novel AS and its association with the situation in question is also associated with neuroplasticity within the evaluator/selector mechanism. A schematic representation of these processes is given in Figure $\mathbf{1}$.

These processes of selection and potentially even de novo establishment of ASs when a novel situation is encountered are according to the REF-model essential mechanisms in the mediation of normal problem solving. What is special about brain injury is that many 


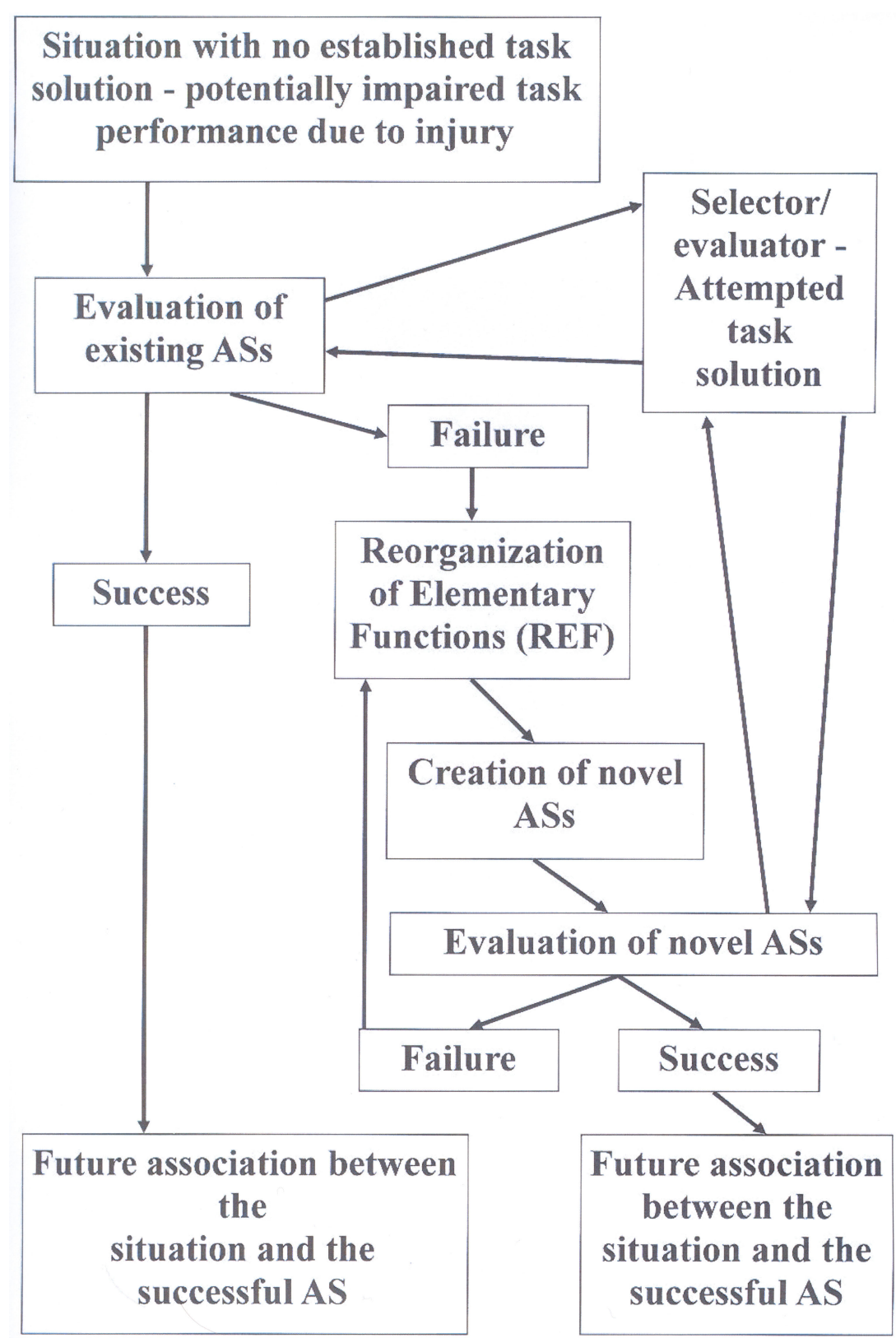

FIGURE 1 | Flow diagram depicting the sequence of events, which according to the REF-model leads to a successful development of a task solution potentially a successful functional recovery after brain injury. These

processes are always associated with plastic modifications within the selector/ evaluator mechanisms. Additional plasticity modifying the connections between the neural substrates of EFs is only expected in case an actual Reorganization of Elementary Functions (REF) process is required. For further details: see the present text as well as Figures 3, 4, and 5 in Mogensen and Malá (2009). situations, for which there used to be an established mechanism of task solution, will after the traumatic event have a status similar to novel situations. The AS, which would normally be activated and ensure an efficient task solution, is no longer available since some of its constituent EFs have been lost to injury. Consequently, there is no immediately available mechanism of task solution. Which leads to the above-described mechanisms of initial search for an appropriate AS. Since multiple ASs might pre-traumatically have been able to allow an efficient task solution, some of these might post-traumatically still be available. If this is the case, the search for an available and appropriate AS will be successful. Alternatively, the REF-process (including backpropagation-based reorganization of interconnectivity between the neural substrates of EFs) is required in order to obtain a satisfactory solution to the task. Viewed in this way, the processes allowing a post-traumatic functionally recovery to take place have mostly if not exclusively evolved as mechanisms mediating problem solving in the intact brain.

In one study addressing the types of neural and cognitive reorganizational processes described by the REF-model (Mogensen et al., 2004) the mechanisms of post-traumatic functional recovery 
of a water maze based allocentric place learning task of the mapping type was addressed in rats subjected to various types of focal brain injury. Lesions of the hippocampus provoked a major functional impairment, which, however, disappeared completely during an approximately 1 month long post-traumatic training period - leaving the animals capable of a task proficiency indistinguishable from that of intact rats. This recovery process depended on contributions from the prefrontal cortex. Relying on partly prefrontal cortical mechanisms, the animals could thus achieve a fully proficient surface phenomenon - task performance. It turned out, however, that even rats subjected to hippocampal lesions as well as removal of the prefrontal cortex were able to achieve an equal - that is: normal proficiency of task performance within a similar period of training. In the absence of both hippocampal and prefrontal contributions to the mediation of task performance, the neural substrate of task solution appeared to depend upon neural mechanisms within the parietal association cortex. In terms of behavioral parameters such as the time and swim distance required to reach the hidden target location, functional recovery mediated by mechanisms within the prefrontal cortex and parietal association cortex, respectively, were of equal proficiency. In the terminology of the REF-model this means that equally proficient ASs can be constructed on the basis of populations of EFs including either prefrontally based EFs or EFs mediated by the parietal association cortex, respectively. Although equally proficient, the ASs relying on prefrontal and parietal mechanisms, respectively, differed with respect to the cognitive mechanisms (as opposed to proficiency) of task solution (as would be expected from the REF-model): While the recovered task solution in animals relying on prefrontal task mediation included cognitive representations of the goal position, such a "knowledge" of the spatial location of the goal appeared to be absent in even fully recovered animals relying on mechanisms within the parietal association cortex (Mogensen et al., 2004).

The situation in which a "complete post-traumatic functional recovery" is encountered as described at the level of surface phenomena, the actual situation is that the post-traumatically selected and potentially established AS is able to allow such a proficiency of task solution that - using standard methods of analysis - the result cannot be distinguished from the pre-traumatic situation. Although each AS is, in fact, associated with a particular way of solving the task, the observable behavior or subjective experience at the level of surface phenomena may in all of these cases be similar enough to be (in a sense wrongly) identified as "the same." And it is this "sameness" of in reality dissimilar surface phenomena that is a crucial aspect of the apparent contradiction between localization and post-traumatic recovery of functions. What is truly localized are the EFs, and when the neural substrate of these basic information processing entities is lost, there is no recovery of the "function" associated with that information processing. In contrast, functional recovery is identified at the level of surface phenomena where highly detailed and special analytical techniques are required in order to discriminate between the phenomena associated with activation of various ASs.

The dynamic reorganizations associated with the REF-process only affect the input/output relationships of the EFs. The actual information processing conducted by the individual EF remains unchanged. It does, however, contribute this information processing within a novel context - within the newly established AS. The situation in which an information processing module continues to perform its previous operations but on a novel input, bears a certain resemblance to some of the plastic processes found in uninjured brains. The somatosensory cortex, which after the amputation of a hand has become "vacant," continues its functional activities - but now operating on information regarding the face or arm (e.g., Yang et al., 1994; Weiss et al., 2000; Karl et al., 2001). Such relative shifts within the somatosensory representations can also be seen after intensive training restricted to part of the body (e.g., Merzenich and Jenkins, 1993; Elbert et al., 1995; Xerri et al., 1996; Münte et al., 2002). These plastic processes within the somatosensory system are not unique. Within the auditory system tonotopic representations undergo plastic changes due to changes in input or experience (e.g., Robertson and Irvine, 1989; Scheich, 1991; Recanzone et al., 1993; Irvine, 2007; Thai-Van et al., 2007). Another obvious parallel is the situation in which a cortical area specialized in analysis of figure orientation within the visual domain can become engaged in apparently similar or at least related information processing on somatosensory information in the blind (Ptito et al., 2005).

Given the highly specialized information processing units of the REF-model - the EFs - this model falls within what is called "Massive Modularity" by for instance Barrett and Kurzban (2006). It should, however, be noted that the modularity of the REF-model is far from identical to the kind of modularity described by Fodor (1983). That radical type of modularity has, however, subsequently been denounced by Fodor (2000) himself.

As emphasized above and by Mogensen and Malá (2009), according to the REF-model apparently the same surface phenomenon may be achieved by activation of a variety of ASs. In the terminology of Price and Friston (Price and Friston, 2002; Friston and Price, 2003) such a situation represents a degeneracy relative to the manifestation of the surface phenomena. In cases where multiple ASs give rise to surface phenomena that cannot be distinguished from each other and that surface phenomenon is then characterized as one "function," such a "function" is degenerate. It should, however, be stressed that according to the REF-model, such a degeneracy is the result of considering multiple surface phenomena, which might by a more or less superficial examination be indistinguishable from each other, "the same" - in spite of the fact that these surface phenomena are in reality different (although perhaps only marginally so). This analysis seems to be in agreement with a number of the examples given by Price and Friston (2002). Degeneracy is by Price and Friston (2002) illustrated by examples in which successful solution of a task (in the terminology of the REF-model: surface phenomenon) can be achieved via activity in separate and potentially not overlapping neural systems. It is recognized that - for instance in case of a linguistic task - the separate systems which are individually able to achieve a successful task solution do perform dissimilar types of information processing and thereby mediate dissimilar types of cognitive analysis. Such systems would in the REF-model be identified as separate ASs. While degeneracy might, thus, apply to the neural and cognitive mechanisms of a particular surface phenomenon, there is according to the REF-model no degeneracy with respect to the substrate of EFs. An EF and its neural substrate are unique and if lost due to injury not replaced. 


\section{IMPLICATION OF THE REORGANIZATIONS OF THE INJURED BRAIN}

When studying brain injured individuals it is important to realize that the result of the recovery process is not - at the neural and more basic cognitive levels - a return to the pre-traumatic situation. Instead it constitutes a novel state of affairs, which has been constructed in an interaction with the environment during the period of rehabilitation. The ASs which form the basis of the posttraumatically observable surface phenomena have been selected and potentially constructed via the interactions between the injured individual and the broadly defined environment. This means that a post-traumatic recovery process can be very situational specific. While the symptoms within a cognitive domain can appear to have disappeared completely when tested in one situation and under certain circumstances, symptoms within the same cognitive domain in the same individual may be evident and for that matter show no signs of recovery when tested under different circumstances.

As has been emphasized elsewhere (e.g., Mogensen, 2003, 2011; Mogensen, in preparation; Overgaard and Mogensen, 2011; Wilms and Mogensen, in preparation) animal models have frequently demonstrated that while one variant (/setup) of a cognitive test is able to reveal a striking level of post-traumatic impairment, another test (/setup), which characterized according to the formal demands of that cognitive test must be considered identical, shows no sign of post-traumatic impairment.

Across all studied species, animals subjected to lesions within the prefrontal cortex or the associated structures such as the prefrontal part of the neostriatum show an impaired performance of the task known as spatial delayed alternation (e.g., Mogensen, 2003; Mogensen et al., 2007, 2008). Nevertheless, even this phenomenon is more situational specific than one would have expected. Mogensen et al. (1987) tested rats subjected to lesions of the prefrontal part of the neostriatum (a lesion which also renders the prefrontal cortex inoperable due to undercutting) in two variants of the spatial delayed alternation task. While the variant administered in a T-maze clearly revealed the expected symptoms, an operant chamber-based version of the task (in spite of fulfilling all the procedural/cognitive demands normally made on such a test setup) showed no sign of post-traumatic impairment. A somewhat related demonstration of the importance of the procedures employed in animal models can be found in a study by Lepore et al. (1985). Cats were tested for their ability to transfer visual discrimination-relevant information from one hemisphere to the other in the absence of the corpus callosum - the main pathway between the two hemispheres. After having acquired the visual discrimination task based on one hemisphere exclusively (information was provided via one eye only in animals with a split optic chiasm), cats subjected to lesions of the corpus callosum were tested for their ability to perform the task with only the contralateral eye open - a situation in which the task performance had to be based on the hemisphere contralateral to the one originally trained. The animals were tested for this ability in two experimental setups: a "Lashley-type" jumping-stand and a traditional (maze-like) two-choice discrimination box. When tested in the discrimination box, the cats did not demonstrate any ability to transfer information between the hemispheres subcallosally, while such an ability was clearly revealed when the test was performed in the jumping-stand.
While animal model-based studies are often able to more clearly demonstrate the procedure and setup-related differences in the degree or even presence of post-traumatic symptoms and posttraumatic recovery, clinical data are frequently of a more anecdotal nature. There are, however, studies in which such phenomena have been scrutinized in patients under controlled circumstances. One example is the results of Wilms and Malá (2010). The background of this study is a fascinating method of rehabilitative training of brain injured patients suffering hemispatial neglect (e.g., Rossetti et al., 1998): the prism adaptation therapy (PAT; e.g., Rossetti et al., 1998; Frassinetti et al., 2002). In this method, the patients are trained in a task requiring them to point (without being able visually to follow their arm during the pointing movement) to targets defined by the therapist - and doing so when wearing prism goggles which diverts the visual field $10^{\circ}$ to the right (the patients are exhibiting a hemispatial neglect of the left hemispace). Normally, the feedback provided to the patient is the sight of the pointing finger at the moment when the pointing movement has been terminated. In most cases, the patient will gradually adapt to the perceptual shift and eventually show an after-effect in the form of a relative shift of the pointing movement - even after the removal of the goggles. In other words, the procedure constitutes an at least partial therapeutic intervention regarding the neglect of the left hemispace. It has been demonstrated that an essential element of the procedure is the feedback regarding the precision of the pointing movements during the training period (e.g., Frassinetti et al., 2002; Serino et al., 2006, 2007; Sarri et al., 2008). In the study by Wilms and Malá (2010) this traditional version of the PAT-procedure was included - and compared directly to a procedure in which the patients pointed to a touch-sensitive computer screen and feedback was provided graphically on the screen rather than via the direct sight of the pointing finger. Surprisingly, in both patients and normal subjects the two procedures differed significantly - the version in which an icon on the computer screen provided the feedback did not lead to any demonstrable after-effect.

Clinically, this specificity of the post-traumatic recovery poses a significant problem with respect to obtaining a therapeutic outcome, which generalizes to for instance the every-day-situations of the patient at home or at the work place. But with respect to studies addressing neural organization and reorganization - and for that matter the neural substrate of consciousness - this specificity may present both problems and promises.

The problems caused by the rather specific reorganizations provoked by a particular post-traumatic training process are primarily related to the (lack of) generality of the conclusions, which can be drawn from studies of a "functionally recovered" patient or animal. The pattern of neural mechanisms (e.g., regional activations seen in neuroimaging studies) and cognitive mechanisms allowing a more or less successful task solution cannot be seen as a more global indication of which parts of the brain are able to "take over" from those lost to injury. Instead, the observable pattern is the result of a specific process, which in principle is only designed to solve the manifestation of the task, which has been trained.

But exactly this specificity may also become a window through which much more detailed information can be obtained. If certain demands are fulfilled, a novel - more difficult and refined - but also more promising type of "localization" research may 
be conducted. Scrutinizing the post-traumatic situation in both patients and experimental animals, such research may elucidate central aspects of the organization and post-traumatic reorganization of the brain - thereby providing a better insight into the neural mechanisms of cognition and consciousness.

And which demands are then to be met by such studies of posttraumatic symptomatology and recovery?

An essential aspect will be a more refined conceptualization of what constitutes a "function" and what is actually obtained during post-traumatic functional recovery. Replacing the old contradiction between localization and recovery of function with the more detailed concepts of for instance the EFs and ASs of the REFmodel can provide a better framework for conceptualization of the results obtained.

But not only conceptually will progress have to be made. Also the methodology of such studies needs refinement. Realizing (like what is indicated by the REF-model) that rehabilitative training obtains a relatively task-specific reorganization rather than a recreation of what has been lost to injury, will have to provoke a different type of research strategy. A recovery process must be systematically addressed across various manifestations of what appear to be the same cognitive task as well as across cognitive domains. For each task it should also (as far as possible) be attempted to include studies utilizing various types of "organic" as well as behavioral/ cognitive "challenges" - as has been suggested by Mogensen and

\section{REFERENCES}

Ansaldo, A. I., and Arguin, M. (2003). The recovery from aphasia depends on both the left and right hemispheres: three longitudinal case studies on the dynamics of language function after aphasia. Brain Lang. 87, 177-178.

Ansaldo, A. I., Arguin, M., and Lecours, A. R. (2002). The contribution of the right cerebral hemisphere to the recovery from aphasia: a single longitudinal case study. Brain Lang. 82, 206-222.

Arvidsson, A., Collin, T., Kirik, D., Kokaia, Z., and Lindvall, O. (2002). Neuronal replacement from endogenous precursors in the adult brain after stroke. Nat. Med. 8, 963-970.

Barrett, H. C., and Kurzban, R. (2006). Modularity in cognition: framing the debate. Psychol. Rev. 113, 628-647.

Bartolomeo, P. (2007). Visual neglect. Curr. Opin. Neurol. 20, 381-386.

Baumgaertner, A., Schraknepper, V., and Saur, D. (2005). Differential recovery of aphasia and apraxia of speech in an adolescent after infarction of the left frontal lobe: longitudinal behavioral and fMRI data. Brain Lang. 95, 211-212.

Berardi, N., Pizzorusso, T., and Maffei, L. (2004). Extracellular matrix and visual cortical plasticity: freeing the synapse. Neuron 44, 905-908.

Berry, M. (1982). Post-injury myelinbreakdown products inhibit axonal growth: an hypothesis to explain the failure of axonal regeneration in the mammalian central nervous system. Bibl. Anat. 23, 1-11.

Brose, K., Bland, K. S., Wang, K. H., Arnott, D., Henzel, W., Goodman, C. S., Tessier-Lavigne, M., and Kidd, T. (1999). Slit proteins bind Robo receptors and have an evolutionarily conserved role in repulsive axon guidance. Cell 96, 795-806.

Buller, D. J., and Hardcastle, V. G. (2000). Evolutionary psychology, meet developmental neurobiology: against promiscuous modularity. Brain Mind 1, 307-325.

Carney, N., Chesnut, R. M., Maynard, H. Mann, N.C., Patterson, P., and Helfand, M. (1999).Effect of cognitive rehabilitation on outcomes for persons with traumatic brain injury: a systematic review. J. Head Trauma Rehabil. 14, 277-307.

Chen, D. F., Jhaveri, S., and Schneider, G. E. (1995). Intrinsic changes in developing retinal neurons result in regenerative failure of their axons. Proc. Natl. Acad. Sci. U.S.A. 92, 7287-7291.

Chen, J., Magavi, S. S., and Macklis, J. D. (2004). Neurogenesis of corticospinal motor neurons extending spinal projections in adult mice. Proc. Natl. Acad. Sci. U.S.A. 101, 16357-16362.

Chen, M.S., Huber, A. B., Van Der Haar, M. E., Frank, M., Schnell, L., Spillmann, A. A., Christ, F., and Schwab, M.E. (2000). Nogo-A is a myelin-associated neurite outgrowth inhibitor and an antigen for monoclonal antibody IN-1. Nature 403, 434-439.

Malá (2009) and Mogensen (2011). Such challenge procedures can provide more detailed insights into the pattern of neural activities mediating a recovered task performance as well as the cognitive strategies allowing a particular surface phenomenon to be achieved.

The plastic nature of the brain - and especially the regionally injured brain - can provide many surprises and frustrations to the neuroscientist trying to understand this most complicated product of nature. Apparently similar tasks provide dissimilar results post-traumatically, and patients who apparently "just" need a precise feedback regarding their pointing errors during rehabilitative training of hemispatial neglect respond differentially in case of a graphic representation on a screen or the actual sight of their finger. These and many more frustrations can in the short term become obstacles to clinical utilization of methods as well as the possibility to reach more global conclusions. But like the apparent contradiction between localization and recovery of function they are also the types of data, which we should cherish and on which we should focus our attention. Because here the dynamic brain is offering us windows through which we may eventually understand the neural mechanisms of cognition - and maybe consciousness.

\section{ACKNOWLEDGMENT}

The present study was supported by a grant from the Danish Research Council.

Choi, J. Y., Lee, K. H., Na, D. L., Byun, H. S., Lee, S. J., Kim, H., Kwon, M. Lee, K.-H., and Kim, B.-T. (2007). Subcortical aphasia after striatocapsular infarction: quantitative analysis of brain perfusion SPECT using statistical parametric mapping and a statistical probabilistic anatomic map. J. Nucl. Med. 48, 194-200.

Coltheart, M. (2001). "Assumptions and methods in cognitive neuropsychology," in The Handbook of Cognitive Neuropsychology, ed. B. Rapp (Philadelphia, PA: Psychology Press), 3-21.

Danckert, J., and Goodale, M. A. (2000). Blindsight: a conscious route to unconscious vision. Curr. Biol. 10, R64-R67.

Del Rio, J. A., and Soriano, E. (2007) Overcoming chondroitin sulphate proteoglycan inhibition of axon growth in the injured brain: lessons from chondroitinase ABC. Curr. Pharmacol. Des. 13, 2485-2492.

Delfs, J. M., Ciaramitaro, V. M., Parry, T. J., and Chesselet, M.-F. (1995) Subthalamic nucleus lesions: widespread effects on changes in gene expression induced by nigrostriatal dopamine depletion in rats. J. Neurosci. 15, 6562-6575.

Elbert, T., Pantev, C., Weinbruch, C. Rockstroh, B., and Taub, E. (1995). Increased cortical representation of the fingers of the left hand in string players. Science 270, 305-307.
Fawcett, J. W., Housden, E., SmithThomas, L., and Meyer, R. L. (1989). The growth of axons in three-dimensional astrocyte cultures. Dev. Biol. 135, 449-458.

Fodor, J. (1983). The Modularity of Mind. Cambridge, MA: MIT Press.

Fodor, J. (2000). The Mind Doesn't Work that Way: The Scope and Limits of ComputationalPsychology. Cambridge, MA: MIT Press.

Frassinetti, F., Angeli, V., Meneghello, F., Avanzi, S., and Ladavas, E. (2002). Long-lasting amelioration of visuospatial neglect by prism adaptation. Brain 125, 608-623.

Friston, K. J., and Price, C. J. (2003). Degeneracy and redundancy in cognitive anatomy. Trends Cogn. Sci. 7, 151-152.

Frost, S. B., Barbay, S., Friel, K. M., Plautz, E. J., and Nudo, R. J. (2003). Reorganization of remote cortical regions after ischemic brain injury: a potential substrate for stroke recovery. J. Neurophysiol. 89, 3205-3214.

Funk, J., Finke, K., Müller, H. J., Preger, R., and Kerkhoff, G. (2010). Systematic biases in the tactile perception of the subjective vertical in patients with unilateral neglect and the influence of upright vs. supine posture. Neuropsychologia 48, 298-308.

Goldberg, J. L., Klassen, M. P., Hua, Y., and Barres, B. A. (2002). Amacrinesignaled loss of intrinsic axon growth 
ability by retinal ganglion cells. Science 296, 1860-1864.

Hagemann, G., Redecker, C., NeumannHaefelin, T., Freund, H.-J., and Witte, O. W. (1998). Increased long-term potentiation in the surround of experimentally induced focal cortical infarction. Ann. Neurol. 44, 255-258.

Harel, N. Y., and Strittmatter, S. M. (2006). Can regenerating axons recapitulate developmental guidance during recovery from spinal cord injury? Nat. Rev. Neurosci. 7, 603-616.

Harris, L. K., Black, R. T., Golden, K. M., Reeves, T. M., Povlishock, J. T., and Phillips, L. L. (2001). Traumatic brain injury-induced changes in gene expression and functional activity of mitochondrial cytochrome $c$ oxidase. J. Neurotrauma 18, 993-1009.

Hermann, D.M., Mies, G., and Hossmann, K.-A. (1999). Biochemical changes and gene expression following traumatic brain injury: role of spreading depression. Restor. Neurol. Neurosci. 14, 103-108.

Hiramoto, M., Hiromi, Y., Giniger, E., and Hotta, Y. (2000). The Drosophila Netrin receptor Frazzled guides axons by controlling Netrin distribution. Nature 406, 886-889.

Irvine, D. R. F. (2007). Auditory cortical plasticity: does it provide evidence for cognitive processing in the auditory cortex? Hear. Res. 229, 158-170.

Karl, A., Birbaumer, N., Lutzenberger, W., Cohen, L. G., and Flor, H. (2001). Reorganization of motor and somatosensory cortex in upper extremity amputees with phantom limb pain. J. Neurosci. 15, 3609-3618.

Kentridge, R. W., Heywood, C. A., and Weiskrantz, L. (1999). Attention without awareness in blindsight. Proc. R. Soc. Lond. B Biol. Sci. 266, 1805-1811.

Keynes, R. J., and Cook, G. M. (1992). Repellent cues in axon guidance. Curr. Opin. Neurobiol. 2, 55-59.

Kleim, J. A., Jones, T. A., and Schallert, T. (2003). Motor enrichment and the induction of plasticity before or after brain injury? Neurochem. Res. 28, 1737-1769.

Koeberle, P. D., and Bahr, M. (2004). Growth and guidance cues for regenerating axons: where have they gone? J. Neurobiol. 59, 162-180.

Krechevsky, I. (1932). "Hypotheses" in rats. Psychol. Rev. 39, 516-532.

Krechevsky, I. (1933). Hereditary nature of "hypotheses". J. Comp. Psychol. 16, 99-116.

Kringelbach, M. L., and Rolls, E. T. (2004). The functional neuroanatomy of the human orbitofrontal cortex: evidence from neuroimaging and neuropsychology. Prog. Neurobiol. 7 , 341-372.
Lane, R. D., Ahern, G. L., Schwartz, G. E., and Kaszniak, A. W. (1997). Is alexithymia the emotional equivalent of blindsight? Biol. Psychiatry 42 834-844.

Leavitt, B. R., Hernit-Grant, C. S., and Macklis, J. D. (1999). Mature astrocytes transform into transitional radial glia within adult mouse neocortex that supports directed migration of transplanted immature neurons. Exp. Neurol. 157, 43-57.

León-Carrión, J., and Machuca-Murga, F. (2001). Spontaneous recovery of cognitive functions after severe brain injury: when are neurocognitive sequelae established? Rev. Esp. Neuropsicol. 3, 58-67.

Lepore, F., Ptito, M., Provencal, C., Bedard, S., and Guillemot, J.-P. (1985). Interhemispheric transfer of visual training in the split-brain cat: effects of the experimental set-up. Can. J. Psychol. 37, 527.

Magavi, S. S., Leavitt, B. R., and Macklis, J. D. (2000). Induction of neurogenesis in the neocortex of adult mice. Nature 405, 951-955.

Marcel, A. J. (1998). Blindsight and shape perception: deficit of visual consciousness or of visual function? Brain 121, 1565-1588.

McGee, A. W., Yang, Y., Fischer, Q. S., Daw, N. W., and Strittmatter, S. M. (2005). Experience-driven plasticity of visual cortex limited by myelin and Nogo receptor. Science 309, 2222-2226.

Meinzer, M., Flaisch, T., Breitenstein, C., Wienbruch, C., Elbert, T., and Rockstroh, B. (2008). Functional rerecruitment of dysfunctional brain areas predicts language recovery in chronic aphasia. Neuroimage 39, 2038-2046.

Merzenich, M. M., and Jenkins, W. M. (1993). Reorganization of cortical representations of the hand following alterations of skin inputs induced by nerve injury, skin island transfers, and experience. J. Hand Therapy 6 , 89-104.

Mogensen, J. (2003). "Animal models in neuroscience," in Handbook of Laboratory Animal Science, 2nd Edn, Vol. II. Animal Models, eds J. Hau and G. L. van Hoosier (Boca Raton, FL: CRC Press LLC), 95-109.

Mogensen, J. (2011). "Animal models in neuroscience," in Handbook of Laboratory Animal Science, 3rd Edn, Vol. II. Animal Models, eds J. Hau and S. Schapiro (Boca Raton, FL: CRC Press LLC) (in press).

Mogensen, J., Boyd, M. H., Nielsen, M. D. Kristensen, R. S., and Malá, H. (2008). Erythropoietin improves spatial delayed alternation in a T-maze in rats subjected to ablation of the prefrontal cortex. Brain Res. Bull. 77, 1-7.
Mogensen, J., Christensen, L. H., Johansson, A., Wörtwein, G., Bang, L. E., and Holm, S. (2002). Place learning in scopolamine treated rats: the roles of distal cues and catecholaminergic mediation. Neurobiol. Learn. Mem. $78,139-166$

Mogensen, J., Hjortkjær, J., Ibervang, K. L., Stedal, K., and Malá, H. (2007). Prefrontal cortex and hippocampus in posttraumatic functional recovery: spatial delayed alternation by rats subjected to transection of the fimbria-fornix and/or ablation of the prefrontal cortex. Brain Res. Bull. $73,86-95$.

Mogensen, J., Iversen, I. H., and Divac, I (1987). Neostriatal lesions impaired rats' delayed alternation performance in a T-maze but not in a two-key operant chamber. Acta Neurobiol. Exp. 47 45-54.

Mogensen, J., Lauritsen, K. T., Elvertorp S., Hasman, A., Moustgaard, A., and Wörtwein, G. (2004). Place learning and object recognition by rats subjected to transection of the fimbria-fornix and/or ablation of the prefrontal cortex. Brain Res. Bull. 63 , 217-236.

Mogensen, J., and Malá, H. (2009). Posttraumatic functional recovery and reorganization in animal models. A theoretical and methodological challenge. Scand. J. Psychol. 50, 561-573.

Mogensen, J., Moustgaard, A., Khan, U., Wörtwein, G., and Nielsen, K. S (2005). Egocentric spatial orientation in a water maze by rats subjected to transection of the fimbria-fornix and/ or ablation of the prefrontal cortex Brain Res. Bull. 65, 41-58.

Mogensen, J., Wörtwein, G., Plenge, P., and Mellerup, E. T. (2003). Serotonin locomotion, exploration, and place recall in the rat. Pharmacol. Biochem. Behav. 75, 381-395.

Monakow, C. V. (1914). Die Lokalisation im Grosshirn und der Abbau der Funktion durch Kortikale Herde. Wiesbaden: Bergmann.

Münte, T. F., Altenmüller, E., and Jäncke, L. (2002). The musician's brain as a model of neuroplasticity. Nat. Rev. Neurosci. 3, 473-478.

Nakatomi, H., Kuriu, T., Okabe, S. Yamamoto, S.-C., Hatano, O. Kawahara, N., Tamura, A., Kirino, T., and Nakafuku, M. (2002). Regeneration of hippocampal pyramidal neurons after ischemic brain injury by recruitment of endogenous neural progenitors. Cell 110, 429-441.

Natsoulas, T. (1997). Blindsight and consciousness. Am. J. Psychol. 110, 1-33.

Norman, D. A., and Shallice, T. (1986) "Attention to action: willed and automatic control of behavior," in Consciousness and Self-Regulation, $\mathrm{Vol}$
4, eds R. J. Davidson, G. E. Schwartz, and D. Shapiro (New York: Plenum Press), 1-18.

Nudo, R. J. (2003). Adaptive plasticity in motor cortex: implications for rehabilitation after brain injury. J. Rehab. Med. 41(Suppl.), 7-10.

Olton, D. S. (1978). "The function of septo-hippocampal connections in spatially organized behaviour," in Functions of the Septo-Hippocampal System, Ciba Foundation Symposium 58 (New York: Elsevier), 327-342.

Overgaard, M., and Mogensen, J. (2011). A framework for the study of multiple realizations: the importance of levels of analysis. Front. Conscious. Res. (in press).

Panksepp, J., and Panksepp, J. B. (2000). The seven sins of evolutionary psychology. Evol. Cogn. 6, 108-131.

Pascual-Leone, A., Bartres-Faz, D., and Keenan, J.P. (1999). Transcranial magnetic stimulation: studying the brainbehaviour relationship by induction of "virtual lesions". Philos. Trans. R. Soc Lond. B 354, 1229-1238.

Pascual-Leone, A., Walsh, V., and Rothwell, J. (2000). Transcranial magnetic stimulation in cognitive neuroscience virtual lesion, chronometry, and functional connectivity. Curr. Opin. Neurobiol. 10, 232-237.

Perani, D., Cappa, S. F., Tettamanti, M., Rosa, M., Scifo, P., Miozzo, A., Basso, A., and Fazio, F. (2003). A fMRI study of word retrieval in aphasia. Brain Lang. 85, 357-368.

Pizzorusso, T., Medini, P., Berardi, N., Chierzi, S., Fawcett, J. W., and Maffei, L. (2002). Reactivation of ocular dominance plasticity in the adult visual cortex. Science 298, 1248-1251.

Polleux, F., Morrow, T., and Ghosh, A. (2000). Semaphorin $3 A$ is a chemoattractant for cortical apical dendrites. Nature 404, 567-573.

Price, C. J., and Friston, K. J. (2002). Degeneracy and cognitive anatomy. Trends Cogn. Sci. 6, 416-421.

Ptito, M., Moesgaard, S. M., Gjedde, A. and Kupers, R. (2005). Cross-modal plasticity revealed by electrotactile stimulation of the tongue in the congenitally blind. Brain 128, 606-614.

Rakic, P. (1971). Guidance of neurons migrating to the fetal monkey neocortex. Brain Res. 33, 471-476.

Rakic, P. (1985). "Mechanisms of neuronal migration in developing cerebellar cortex," in Molecular Basis of Neural Development, eds G. M. Edelman, W. M. Cowan, and E. Gull (New York: Wiley), 139-160.

Ramachandran, V. S., and Blakeslee, S. (1998). Phantoms in the Brain: Probing the Mysteries of the Human Mind. New York: William Morrow. 
Rao, V. L. R., Dhodda, V. K., Song, G., Bowen, K. K., and Dempsey, R. J. (2003). Traumatic brain injury-induced acute gene expression changes in rat cerebral cortex identified by GeneChip analysis. J. Neurosci. Res. 71, 208-219.

Recanzone, G. H., Schreiner, C. E., and Merzenich, M. M. (1993). Plasticity in the frequency representation of primary auditory cortex following discrimination training in adult owl monkeys. J. Neurosci. 13, 87-103.

Robertson, D., and Irvine, D. R. F. (1989). Plasticity of frequency organization in auditory cortex of guinea pigs with partial unilateral deafness. J. Comp. Neurol. 282, 456-471.

Rohling, M. L., Faust, M. E., Beverly, B., and Demakis, G. (2009). Effectiveness of cognitive rehabilitation following acquired brain injury: a meta-analytic re-examination of Cicerone et al.'s (2000, 2005) systematic reviews. Neuropsychology 23, 20-39.

Rossetti, Y., Rode, G., Pisella, L., Farne, A., Li, L., Boisson, D., and Perenin, M.-T. (1998). Prism adaptation to a rightward optical deviation rehabilitates left hemispatial neglect. Nature 395, 166-169.

Rumelhart, D., and McClelland, J. (1986). Parallel Distributed Processing. Cambridge, MA: MIT Press.

Sarri, M., Greenwood, R., Kalra, L., Papps, B., Husain, M., and Driver, J. (2008). Prism adaptation aftereffects in stroke patients with spatial neglect: pathological effects on subjective straight ahead but not visual open-loop pointing. Neuropsychologia 46, 1069-1080.

Schäfer, R., Dehn, D., Burbach, G. J., and Deller, T. (2008). Differential regulation of chondroitin sulfate proteoglycan mRNAs in the denervated rat fascia dentata after unilateral entorhinal cortex lesion. Neurosci. Lett. 439, 61-69.

Scharff, C., Kirn, J. R., Grossman, M., Macklis, J. D., and Nottebohm, G. (2000). Targeted neuronal death affects neuronal replacement and vocal behavior in adult songbirds. Neuron 25, 481-492.

Scheich, H. (1991). Auditory cortex: comparative aspects of maps and plasticity. Curr. Opin. Neurobiol. 1, 236-247.

Schindler, I., Clavagnier, S., Karnath, H.O., Derex, L., and Perenin, M.-T. (2006). A common basis for visual and tactile exploration deficits in spatial neglect? Neuropsychologia 44, 1444-1451.

Schwab, M. E., and Thoenen, H. (1985). Dissociated neurons regenerate into sciatic but not optic nerve explants in culture irrespective of neurotrophic factors. J. Neurosci. 5, 2415-2423.

Selnes, O. A. (2001). "A historical overview of contributions from the study of deficits," in The Handbook of Cognitive Neuropsychology, ed. B. Rapp (Philadelphia, PA: Psychology Press) 23-41.

Serino, A., Angeli, V., Frassinetti, F., and Ladavas, E. (2006). Mechanisms underlying neglect recovery after prism adaptation. Neuropsychologia 44, 1068-1078.

Serino, A., Bonifazi, S., Pierfederici, L., and Ladavas, E. (2007). Neglect treatment by prism adaptation: what recovers and for how long. Neuropsychol. Rehabil. 17, 657-687.

Silvanto, J., Cowey, A., and Walsh, V. (2008). Inducing conscious perception of colour in blindsight. Curr. Biol. 18, R950-R951.

Specht, K., Zahn, R., Willmes, K., Weis, S., Holtel, C., Krause, B. J., Herzog, H., and Huber, W. (2009). Joint independent component analysis of structural and functional images reveals complex patterns of functional reorganisation in stroke aphasia. Neuroimage 47 , 2057-2063.

Szaflarski, J. P., Eaton, K., Ball, A. L., Banks, C., Vannest, J., Allendorfer, J. B., Page, S., and Holland, S. K. (2010). Poststroke aphasia recovery assessed with functional magnetic resonance imaging and a picture identification task. J. Stroke Cerebrovasc. Dis. 1-10. (published online August 2010).

Thai-Van, H., Micheyl, C., Norena, A., Veuillet, E., Gabriel, D., and Collet, L. (2007). Enhanced frequency discrimination in hearing-impaired individuals: a review of perceptual correlates of central neural plasticity induced by cochlear damage. Hear. Res. 233 , 14-22.

Thomas, C., Altenmüller, E., Marckmann, G., Kahrs, J., and Dichgans, J. (1997). Language processing in aphasia: changes in lateralization patterns during recovery reflect cerebral plasticity in adults. Electroencephalogr. Clin. Neurophysiol. 102, 86-97.

Thulborn, K. R., Carpenter, P. A., and Just, M. A. (1999). Plasticity of language-related brain function during recovery from stroke. Stroke 30 749-754.

Walsh, V., and Cowey, A. (2000). Transcranial magnetic stimulation and cognitive neuroscience. Nat. Rev Neurosci. 1, 73-79.

Weiss, T., Miltner, W. H. R., Huonker, R. Friedel, R., Schmidt, I., and Taub, E. (2000). Rapid functional plasticity of the somatosensory cortex after finger amputation. Exp. Brain Res. 134 199-203.

Werbos, P. J. (1994). The Roots of Backpropagation: From Ordered Derivatives to Neural Networks and Political Forecasting. New York: John Wiley \& Sons.
Wilms, I., and Malá, H. (2010). Indirect versus direct feedback in computerbased Prism Adaptation Therapy. Neuropsychol. Rehabil. 20, 830-853.

Witte, O.W. (1998). Lesion-induced plasticity as a potential mechanism for recovery and rehabilitative training. Curr. Opin. Neurol. 11, 655-662.

Xerri, C., Coq, J., Merzenich, M., and Jenkins, W. (1996). Experienceinduced plasticity of cutaneous maps in the primary somatosensory cortex of adult monkeys and rats. J. Physiol. 90, 277-287.

Yang, T. T., Gallen, C. C., Ramachandran, V. S., Cobb, S., Schwartz, B. J., and Bloom, F. E. (1994). Noninvasive detection of cerebral plasticity in adult human somatosensory cortex. Neuroreport 5, 701-704.

Conflict of Interest Statement: The author declares that the research was conducted in the absence of any commercial or financial relationships that could be construed as a potential conflict of interest.

Received: 03 October 2010; accepted: 05 January 2011; published online: 26 January 2011.

Citation: Mogensen J (2011) Reorganization of the injured brain: implications for studies of the neural substrate of cognition. Front. Psychology 2:7. doi: 10.3389/ fpsyg.2011.00007

This article was submitted to Frontiers in Consciousness Research, a specialty of Frontiers in Psychology.

Copyright () 2011 Mogensen. This is an open-access article subject to an exclusive license agreement between the authors and Frontiers Media SA, which permits unrestricted use, distribution, and reproduction in any medium, provided the original authors and source are credited. 